\title{
Renal failure secondary to pelvic organe prolapse: a diagnosis not to be missed
}

\begin{abstract}
Pelvic organ prolapse (POP) is a common condition in multiparous elderly women and can be associated with hydronephrosis, with or without renal impairment. We present the case of a neglected uterine prolapsed in a 65year's old patient resulting in acute renal failure. Authors explain the possible renal failure mechanisms related to POP, and enhance on the importance of early diagnosis and appropriate treatment to prevent irreversible renal damage.
\end{abstract}

Keywords: pelvic organ prolapse, renal failure, hydronephrosis
Volume I Issue 2 - 2014

\author{
Saad Benkirane,' Fahd Khalil, ${ }^{2}$ Hanane Saadi,' \\ Ahmed Mimouni' \\ 'Department of gynecology, Mohammed the sixth university \\ hospital, Morocco \\ 'Urology department, Mohammed the sixth university hospital, \\ Morocco
}

Correspondence: Fahd Khalil, Urology department, Mohammed the sixth university hospital, Morocco, Email drf.khalil@hotmail.fr

Received: October 19,2014 | Published: November 27, 2014

\section{Introduction}

Pelvic organ prolapse (POP) is a common condition in multiparous elderly women and can be associated with hydronephrosis, with or without renal impairment. ${ }^{1}$ The renal dysfunction is globally related to the degree of pelvic prolapsed, and its consequences ranges from acute to chronic renal failure and may also lead to end stage renal failure. ${ }^{2}$ In this report, we present the case of a neglected uterine prolapsed in a 65year's old patient resulting in acute renal failure.

\section{Case report}

A 65year's old diabetic women with three vaginal deliveries presented with externalized genital prolapse evolving since two years, with dysuria, painful micturition, and intermittent urinary retention. There was no urinary incontinence and the clinical examination found a genital grade IV (Baden and walker classification) prolapsed (Figure 1). Ultrasonography was performed showing bilateral ureteral dilation with thinned renal cortex especially in the right side $\mu$ and a significant post voiding residual (Figure 2). Blood creatinine was $34 \mu \mathrm{mol} / \mathrm{ml}$ and urea at $0.89 \mathrm{~g} / \mathrm{l}$. Urine culture revealed the presence of Escherichia coli, and the cervico-uterine smear was normal. Antibiotic treatment was followed by a vaginal hysterectomy with bladder placation (Figure 3). We associated a spinofixation as the Richter technique with the levator ani myorraphy. Two months later, the patient related a significant clinical improvement with disappearance of urine retention episodes and dysuria and normalization of the renal function. The ultrasonography showed a regression of hydronephrosis and good renal parenchyma.

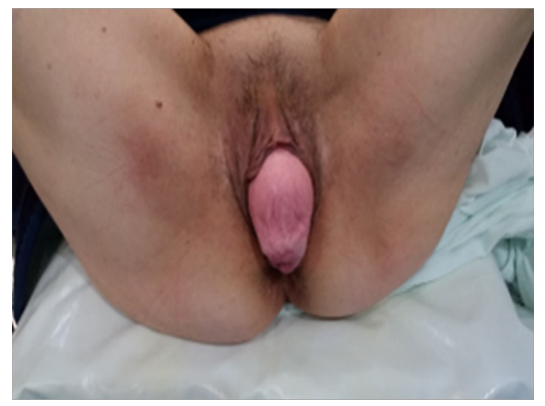

Figure I Externalized grade IV genital prolapsed.

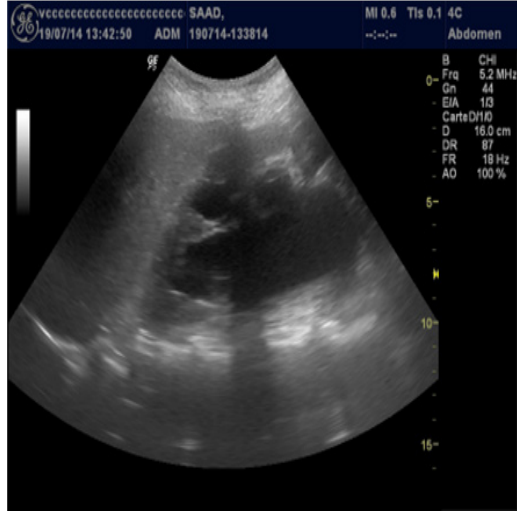

Figure 2 Ultrasonography showing the right kidney hydronephrosis.

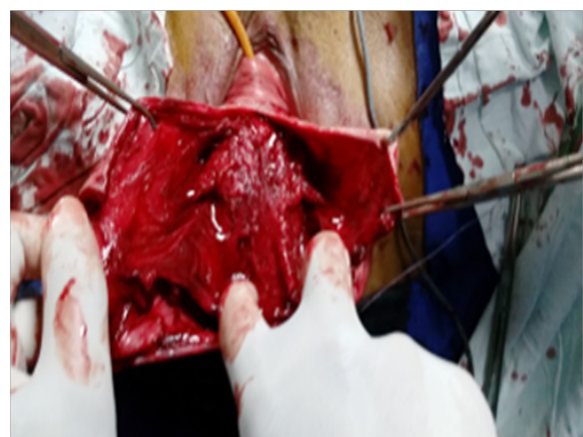

Figure 3 Operative view showing the caudal displacement of the bladder trigone and the ureters's compression between the uterus and the medial borders of the genital hiatus.

\section{Discussion}

The prevalence of hydronephrosis with POP varies from $7 \%$ to $17 \%$ in patients undergoing surgery for pelvic disturbance. ${ }^{1,2}$ The degree of hydronephrosis increases with spoilage grade of the POP. ${ }^{3,4}$ It is much lower with post-hysterectomy vaginal vault prolapse than with actual uterine prolapse. ${ }^{2}$ This complication of POP is uncommon, but reversible, if detected early and treated appropriately. Hydronephrosis associated with POP was first reported in 1824 by Fiorep. ${ }^{2}$ Since that time several investigators have studied this relationship. ${ }^{5}$ One of the 
major complications of pelvic organ prolapse is disturbed drainage of ureters due to procidentia. ${ }^{4}$ The reported incidence of urinary tract obstruction is $5 \%$ for first-degree prolapse and about $40 \%$ for procidentia. ${ }^{6}$ A possible mechanism of renal failure in the presence of POP may be a compression of the ureters by the uterine blood vessels, the levator ani sling, or even the uterine fundus, and the prolapse might cause obstruction of the lower ureters. In addition, the bladder drainage may be disturbed by bladder outlet obstruction. ${ }^{7}$ The caudal displacement of the bladder trigone results in compression of the ureters between the uterus and the medial borders of the genital hiatus. ${ }^{8}$ In this case report, we noted a total synchronous prolapse of the bladder trigone with the uterus, so as the hysterectomy corrected the elongation of both ureters and improves the renal function.

\section{Conclusion}

Genital prolapse is a common and usually benign condition especially among aged and multiparous women. However, it can causes severe complications such as hydronephrosis, urinary tract infections, or acute renal failure which can advanced to an end stage if neglected. We enhance on the importance of early diagnosis and appropriate treatment to prevent irreversible renal damage.

\section{Acknowledgements}

None.

\section{Conflict of interest}

The author declares no conflict of interest.

\section{References}

1. Gemer O, Bergman M, Segal S. Prevalence of hydronephrosis in patients with genital prolapse. Eur J Obstet Gynecol Reprod Biol. 1999;86(1):11-13.

2. Beverly CM, Walters MD, Weber AM, et al. Prevalence of hydronephrosis in patients undergoing surgery for pelvic organ prolapse. Obstet Gynecol. 1997;90(1):37-41.

3. Chuang FR, Lee $\mathrm{CH}$, Chen $\mathrm{CS}$, et al. Bilateral moderate hydroureteronephrosis due to uterine prolapse: two case reports and review of the literature. Ren Fail. 2003;25(5):879-884.

4. Rodriguez Alonso A, Gonzalez Blanco A, Cachay Ayala ME, et al. Obstructive anuria secondary to uterine prolapse. Actas Urol Esp. 2002;26(9):703-707.

5. Brettauer J, Ruben IC. Hydroureter and Hydronephrosis: A frequent secondary finding in cases of prolapsed of uterus and bladder. Am Journal of Obstetrics and Gynecology. 1923;6:696-709.

6. Stavropoulos NJ, Makrychoritis C, Deliveliotis C, et al. Uterine prolapse and urinary tract obstruction. Acta Urol Belg. 1995;63(4):37-38.

7. Yanik FF, Akpolat T, Kocak I. Acute renal failure-an unusual consequence of uterine prolapse. Nephrol Dial Transplant. 1998;13(10):2648-2650.

8. Costantini E, Lazzeri M, Mearini L, et al. Hydronephrosis and pelvic organ prolapse. Urology. 2009;73(2):263-267. 